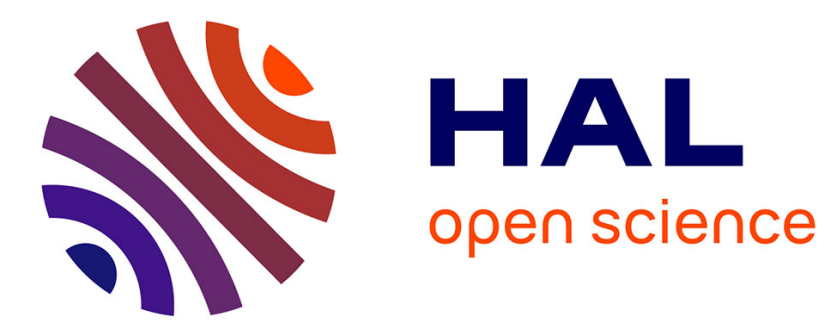

\title{
Median filtering in multispectral filter array demosaicking
}

Xingbo Wang, Jean-Baptiste Thomas, Jon Yngve Hardeberg, Pierre Gouton

\section{To cite this version:}

Xingbo Wang, Jean-Baptiste Thomas, Jon Yngve Hardeberg, Pierre Gouton. Median filtering in multispectral filter array demosaicking. Digital Photography IX, Feb 2013, Burlingame, United States.

Digital Photography IX, 86600E, 10.1117/12.2005256 . hal-00847768

\section{HAL Id: hal-00847768 \\ https://hal.science/hal-00847768}

Submitted on 24 Jul 2013

HAL is a multi-disciplinary open access archive for the deposit and dissemination of scientific research documents, whether they are published or not. The documents may come from teaching and research institutions in France or abroad, or from public or private research centers.
L'archive ouverte pluridisciplinaire HAL, est destinée au dépôt et à la diffusion de documents scientifiques de niveau recherche, publiés ou non, émanant des établissements d'enseignement et de recherche français ou étrangers, des laboratoires publics ou privés. 


\title{
Median filtering in multispectral filter array demosaicking
}

\author{
Xingbo Wang ${ }^{a, b}$, Jean-Baptiste Thomas $^{a}$, Jon Yngve Hardeberg ${ }^{b}$, Pierre Gouton ${ }^{a}$ \\ ${ }^{a}$ Université de Bourgogne, Dijon, France; \\ ${ }^{b}$ Gjøvik University College, Gjøvik, Norway
}

\begin{abstract}
Inspired by the concept of the colour filter array (CFA), the research community has shown much interest in adapting the idea of CFA to the multispectral domain, producing multispectral filter arrays (MSFAs). In addition to newly devised methods of MSFA demosaicking, there exists a wide spectrum of methods developed for CFA. Among others, some vector based operations can be adapted naturally for multispectral purposes. In this paper, we focused on studying two vector based median filtering methods in the context of MSFA demosaicking. One solves demosaicking problems by means of vector median filters, and the other applies median filtering to the demosaicked image in spherical space as a subsequent refinement process to reduce artefacts introduced by demosaicking. To evaluate the performance of these measures, a tool kit was constructed with the capability of mosaicking, demosaicking and quality assessment. The experimental results demonstrated that the vector median filtering performed less well for natural images except black and white images, however the refinement step reduced the reproduction error numerically in most cases. This proved the feasibility of extending CFA demosaicking into MSFA domain.
\end{abstract}

Keywords: Demosaicking, multispectral, median filtering, n-sphere, colour filter array, spectral filter array

\section{INTRODUCTION}

Since the invention of the colour filter array (CFA) by Bayer ${ }^{1}$ in 1976, it has resulted in enormous success in a wide spectrum of applications ranging from consumer and professional photography, scientific research, all the way to industrial operations, thanks to its capability of simultaneous and in-plane colour separation that permits the acquisition of a colour image by means of one sensor at one exposure. Not only does a CFA ensure the cost effectiveness and compactness of an imaging apparatus, but it also provides high performance and great reliability in terms of colour registration. Nevertheless the advantages mentioned above are gained at the expense of reduced spatial image resolution due to the intrinsic property of CFAs that there is merely one type of filter at each particular location of the array. In practice one photosensitive element on the sensor usually corresponds to one filter in a CFA, that is, no more than one spectral channel is sensed by each pixel. Therefore certain post processing becomes necessary to recover the colour to a certain extent, and this is called demosaicking.

The great benefits brought by a CFA have also stimulated academic and practical interest among research community as a promising and compromising solution to multispectral image acquisition. To that end, key elements of a CFA based imaging system have to be re-examined. In recent years, sustained research effort has gone into designing multispectral filter arrays (MSFAs) and developing associated demosaicking algorithms. The first notable work in this regard was conducted by Miao et al. ${ }^{2}$ who developed a generic binary tree based generation method of MSFA spatial arrangement starting from a chequerboard pattern, and further proposed a generic MSFA demosaicking method following the same binary tree structure. ${ }^{3}$ To the best of our knowledge, these are the first systematic attempts at MSFA design and demosaicking. This was recently utilised for the further evaluation of the spatial arrangement of filters by Shrestha et al. ${ }^{4}$

Among a wide spectrum of algorithms proposed for CFA demosaicking in the last decades, some of them might be able to be adapted to MSFA demosaicking due to the intrinsic properties that CFA and MSFA share. A

Further author information: (Send correspondence to Xingbo Wang)

Xingbo Wang: E-mail: xingbo.wang@hig.no, Telephone: +4761 135395

Jean-Baptiste Thomas: E-mail: jean-baptiste.thomas@u-bourgogne.fr

Jon Yngve Hardeberg: E-mail: jon.hardeberg@hig.no

Pierre Gouton: E-mail: pierre.gouton@u-bourgogne.fr 
beneficial viewpoint is considering each pixel in an image as a vector, and a normal trichromatic image constitutes a three-dimensional vector space. Clearly this concept is compatible with multispectral images, and so are the demosaicking methods based on that. For instance, a CFA demosaicking method that applies vector median filters to the pseudo-pixels formed in a given neighbourhood, was devised by Gupta and Chen. ${ }^{5}$ To reduce the artefacts introduced by demosaicking, such as false colours and degraded edges, Keren and Osadchy ${ }^{6}$ presented a post-processing step that transforms each colour vector in a demosaicked image into an n-sphere and applies median filtering to the spherical coordinates, for the sake of the preservation of luminance discontinuities and the reduction of irregular chromatic variation among neighbouring pixels. As shown in respective literature, both methods give rise to satisfactory results despite their simplicity. As a consequence, we determined to extend and experiment with these two approaches in this paper.

The paper is organised as follows. The two aforementioned methods are described in Section 2 , followed by the procedures and conditions of the experiments presented in Section 3. The primary results are demonstrated and discussed in Section 4. Section 5 draws some conclusions.

\section{METHODS}

\subsection{Vector median filtering in MSFA demosaicking}

In itself, vector median operation originally advanced by Astola et al. ${ }^{7}$ is at the core of the idea detailed in Gupta and Chen. ${ }^{5}$ Different from median filters, vector median is specifically designed for vector-valued signals, for instance, multispectral and colour images. It reduces to the scalar median when the inputs are one-dimensional vectors, and possesses similar properties to scalar median filters, such as the capability of smoothing noisy data while retaining sharp edges. Another basic property of the vector median, inherited from the median filter, is that it does not introduce any new values that do not exist in the input. Therefore the output of the vector median filter must be one of the input vectors. that

According to Definition 1 provided by Astola et al. ${ }^{7}$ the vector median $V M_{\mathrm{L} 2}$ of $\underline{x}_{1}, \ldots, \underline{x}_{N}$ is $\underline{x}_{V M}$ such

$$
\underline{x}_{V M} \in\left\{\underline{x}_{i} \mid i=1, \ldots, N\right\}
$$

and for all $j=1, \ldots, N$

$$
\sum_{i=1}^{N}\left\|\underline{x}_{V M}-\underline{x}_{i}\right\|_{2} \leq \sum_{i=1}^{N}\left\|\underline{x}_{j}-\underline{x}_{i}\right\|_{2}
$$

where \|\|$_{2}$ denotes the $L^{2}$-norm*.

To take advantage of vector median for demosaicking, multiple input vectors are necessary. Gupta and Chen ${ }^{5}$ propose a concept of pseudo-pixel by grouping neighbouring red, green and blue pixels within a given neighbourhood. This leads to quite a few vectors representing all possible pseudo-pixels, each of which consists of three types of pixels connected horizontally or vertically in a mosaicked image.

Taking multispectral image into consideration, we extended this idea from two aspects. Firstly, our implementation associated the size of neighbourhood where the pseudo-pixels are formed with the dimension of a moxel, a mosaic element corresponding to an elementary filter mosaic repeated across an MSFA. Figure 1a shows an example of a moxel when the filter array is composed of four types of filters labelled by the numbers. Figure $1 \mathrm{~b}$ and $1 \mathrm{c}$ demonstrate two neighbourhood dimensions, namely, $(2(n-1)+1) \times(2(m-1)+1)$ and $(2 n+1) \times(2 m+1)$, where $n$ and $m$ denote the number of rows and columns of the moxel. As can be seen, in the larger neighbourhood, there is a rectangular occurrence of 8 neighbouring pixels bearing the same label as the pixel in question situated in the centre. Secondly, in the original work, ${ }^{5}$ only horizontally and vertically connected pixels may be grouped as pseudo-pixels, whereas in this work diagonally connected pixels were also considered. Figure 2a illustrates a case where four pixels connected horizontally and vertically are grouped into one pseudo-pixel, while Figure $2 \mathrm{~b}$ displays a pseudo-pixel formed by pixels connected both horizontally and diagonally.

${ }^{*}$ For a real number $p \geq 1$, the $\boldsymbol{L}^{p}$-norm of $x$ is defined by $\|x\|_{p}=\left(\left|x_{1}\right|^{p}+\left|x_{2}\right|^{p}+\cdots+\left|x_{n}\right|^{p}\right)^{\frac{1}{p}}$. 
Astola et al. ${ }^{7}$ refers to a straightforward algorithm to find the vector median of $\underline{x}_{1}, \ldots, \underline{x}_{N}$ as follows, which was adopted in this work.

a) For each vector $\underline{x}_{i}$, compute the distances to all the other vectors using either the $L^{1}$-norm or the $L^{2}$-norm and add them together, resulting in

$$
S_{i}=\sum_{j=1}^{N}\left\|\underline{x}_{i}-\underline{x}_{j}\right\|, \quad i=1, \ldots, N .
$$

b) Find $\min$ such that $S_{\min }$ is the minimum of $S_{i}$.

c) The vector median is $\underline{x}_{\min }$.

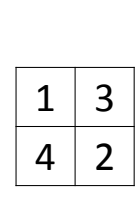

(a) Example of a moxel

\begin{tabular}{|l|l|l|}
\hline 1 & 3 & 1 \\
\hline 4 & 2 & 4 \\
\hline 1 & 3 & 1 \\
\hline
\end{tabular}

(b) Example of small neighbourhood

\begin{tabular}{|l|l|l|l|l|}
\hline 2 & 4 & 2 & 4 & 2 \\
\hline 3 & 1 & 3 & 1 & 3 \\
\hline 2 & 4 & 2 & 4 & 2 \\
\hline 3 & 1 & 3 & 1 & 3 \\
\hline 2 & 4 & 2 & 4 & 2 \\
\hline
\end{tabular}

Figure 1: Moxel and neighbourhood

\begin{tabular}{|r|r|r|r|r|}
\hline 2 & 4 & 2 & 4 & 2 \\
\hline 3 & 1 & 3 & 1 & 3 \\
\hline 2 & 4 & 2 & 4 & 2 \\
\hline 3 & 1 & 3 & 1 & 3 \\
\hline 2 & 4 & 2 & 4 & 2 \\
\hline
\end{tabular}

(a) A pseudo-pixel formed by rectangularly connected pixels

\begin{tabular}{|l|l|l|l|l|}
\hline 2 & 4 & 2 & 4 & 2 \\
\hline 3 & 1 & 3 & 1 & 3 \\
\hline 2 & 4 & 2 & 4 & 2 \\
\hline 3 & 1 & 3 & 1 & 3 \\
\hline 2 & 4 & 2 & 4 & 2 \\
\hline
\end{tabular}

(b) A pseudo-pixel formed by diagonally connected pixels

Figure 2: Formation of pseudo-pixels

\subsection{Median filtering in $n$-sphere as a refinement step}

As demosaicking is an operation of estimation, most methods fail in some situations of one kind or another which often results in false colour artefacts such as blurs and zips. As known, median filtering is effective at correcting such defects. As described in the original paper, ${ }^{6}$ median filtering in spherical space may be used as a post processing step subsequent to demosaicking. However, the median operation should be applied to angular components of the vectors, whilst the luminance related radius should be kept unmodified, so as to keep the luminance to some extent. We followed the principle and carried out the procedure by extending spherical space and three-dimensional Euclidean space to n-sphere and n-dimensional Euclidean space respectively.

To be specific, four steps were conducted in succession as follows.

a) Each pixel is represented by a vector $\underline{x}=\left\{\underline{x}_{1}, \ldots, \underline{x}_{n}\right\}$ in an $\mathrm{n}$-dimensional Euclidean space, where $\mathrm{n}$ denotes the number of spectral bands of the demosaicked multispectral image. 
b) Each vector $\underline{x}$ is transformed to $\underline{y}=\left\{r, \phi_{1}, \ldots, \phi_{n-1}\right\}$ in the $\mathrm{n}$-sphere according to Equation 4 .

c) Two-dimensional median filtering is applied one by one among the $n-1$ angular planes $\left\{\phi_{1}, \ldots, \phi_{n-1}\right\}$, whilst the radial plane $r$ remains unchanged.

d) Each vector is transformed back to $\underline{\hat{x}}=\left\{\underline{\hat{x}}_{1}, \ldots, \underline{\hat{x}}_{n}\right\}$ in the n-dimensional Euclidean space according to Equation 5.

$$
\begin{gathered}
r=\sqrt{\underline{x}_{n}^{2}+\underline{x}_{n-1}^{2}+\cdots+\underline{x}_{2}^{2}+\underline{x}_{1}^{2}}, \\
\phi_{1}=\operatorname{arccot} \frac{\underline{x}_{1}}{\sqrt{\underline{x}_{n}^{2}+\underline{x}_{n-1}^{2}+\cdots+\underline{x}_{2}^{2}}}, \\
\phi_{2}=\operatorname{arccot} \frac{\underline{x}_{2}}{\sqrt{\underline{x}_{n}^{2}+\underline{x}_{n-1}^{2}+\cdots+\underline{x}_{3}^{2}}}, \\
\vdots \\
\phi_{n-2}=\operatorname{arccot} \frac{\underline{x}_{n-2}}{\sqrt{\underline{x}_{n}^{2}+\underline{x}_{n-1}^{2}}}, \\
\phi_{n-1}=2 \operatorname{arccot} \frac{\sqrt{\underline{x}_{n}^{2}+\underline{x}_{n-1}^{2}}+\underline{x}_{n-1}}{\underline{x}_{n}} . \\
\underline{\hat{x}}_{1}=r \cos \left(\phi_{1}\right), \\
\underline{\hat{x}}_{2}=r \sin \left(\phi_{1}\right) \cos \left(\phi_{2}\right), \\
\underline{\hat{x}}_{3}=r \sin \left(\phi_{1}\right) \sin \left(\phi_{2}\right) \cos \left(\phi_{3}\right), \\
\vdots \\
\underline{\hat{x}}_{n-1}=r \sin \left(\phi_{1}\right) \cdots \sin \left(\phi_{n-2}\right) \cos \left(\phi_{n-1}\right), \\
\underline{\hat{x}}_{n}=r \sin \left(\phi_{1}\right) \cdots \sin \left(\phi_{n-2}\right) \sin \left(\phi_{n-1}\right) .
\end{gathered}
$$

\subsection{Test images}

\section{EXPERIMENT}

Eight hyperspectral reflectance images acquired by Foster et al. ${ }^{8}$ were used as test samples in this study. The test set consisted of a mixture of rural scenes containing rocks, trees, leaves, grass, and earth and urban scenes. Scenes were illuminated by direct sunlight in clear or almost clear sky. The spectral span ranges over 400-720 $\mathrm{nm}$ in $10 \mathrm{~nm}$ steps yielding 33 bands, while scene 5 has only 32 bands due to the noisy $720 \mathrm{~nm}$ band. For fair comparisons and because of the little information contained in $720 \mathrm{~nm}$ channel, it was removed from all images yielding 8 hyperspectral images of 32 bands. In addition, one artificial pattern that includes one circle and one line and resembles the first publicly broadcast test card, was also created and employed as the $9^{\text {th }}$ test image. It has 32 bands as well, but only two reflectance values, namely, 0 and 1 . Figure 3 provides an overview on the 9 images. 


\subsection{Procedure}

To conduct the experiments, we decided on a set of algorithms and parameters, as described below.

a) Images were rendered with the illuminant of CIE D65;

b) In each case, spectral transmittances were determined so that each of them had a regular Gaussian shape and the centres of them were evenly distributed over the pertinent spectrum with the overlapping point located at one sigma, as shown in Figure 4;

c) Three filter array patterns were chosen, Bayer type 3-band setup, 4-band setup in form of $2 \times 2$ moxel and 8-band in form of $4 \times 4$ moxel, the moxels were repeated across the whole image;

d) The filter arrays were executed in accordance with the binary tree approach proposed by Miao et al. ${ }^{2}$ a perfect binary tree to be exact, with two levels and three levels corresponding to 4-band and 8-band arrangements respectively, as indicated in Figure 5;

e) Two demosaicking algorithms, bilinear interpolation and Miao et al.'s binary tree based progressive demosaicking, ${ }^{3}$ were implemented and compared with vector median technique;

f) In the case of 3-band setup, vector median was conducted in accordance with the parameter specified by Gupta and Chen, ${ }^{5}$ whilst in case of 4-band and 8-band setup, two types of neighbourhood (small and large) and two ways of connectivities for forming pseudo-pixels were combined, giving rise to 6 groups of parameters;

g) Median filtering in n-sphere was used as a refinement step in combination with all three demosaicking algorithms, and the size of the window was fixed;

h) To visualise the images, the original hyperspectral images were transformed into sRGB colour space, and the demosaicked multispectral images were first restored to hyperspectral images with a spectrum reconstruction method exploiting a priori knowledge of the imaged objects ${ }^{9}$ and then rendered to sRGB.

i) In addition to subjective observations, evaluation of the performance was carried out by means of PSNR computed between the original and reproduced multispectral images, so as to avoid the error introduced by the spectrum reconstruction.

j) The way pseudo-pixels are formed resulted in a considerable amount of pseudo-pixels, 4600 at most in the experiment, which makes vector median filtering process computationally expensive. However it is highly parallelisable, as the neighbourhood of any pixel does not depend on another, and 4 cores were utilised to run 4 demosaicking threads in parallel.

\section{RESULTS}

Table 1 compares the performance of the three demosaicking algorithms in various conditions by means of PSNR. The best results are in bold, while results in italic type correspond to the worst ones. It can be seen that,

a) the PSNRs decrease with the increase of number of filters;

b) Among the three demosaicking algorithms, the binary tree approach, in most cases, gives rise to better results than bilinear interpolation does. The PSNRs yielded by vector median are generally much lower than those of the other two methods, except for Image 9;

c) In extreme conditions, such as binary images akin to Image 9, vector median yields both the best and worst results, since it may select a vector that represents an original pixel value in the image or pick a bizarrely formed vector. The former results in the best results, whereas the latter leads to the worst ones.

d) A large neighbourhood is not beneficial to the vector median in general, nevertheless, it improves the performance significantly when pseudo-pixels do not have to pass through the centre pixel in question in case of the 4-band set-up; 


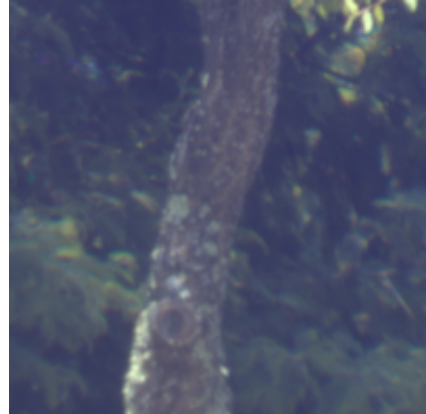

Image 1

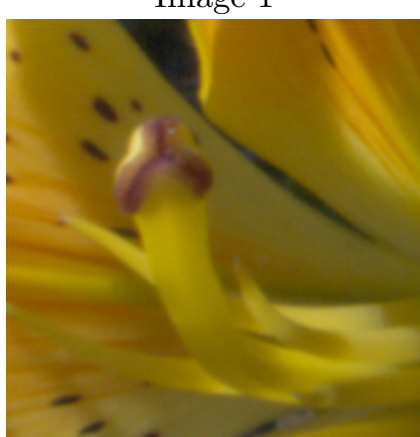

Image 4

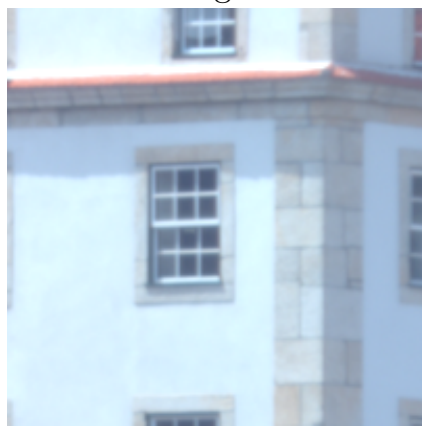

Image 7

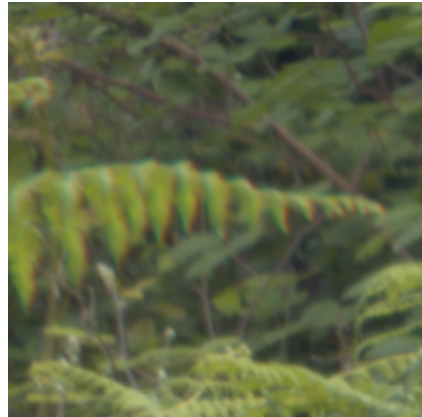

Image 2

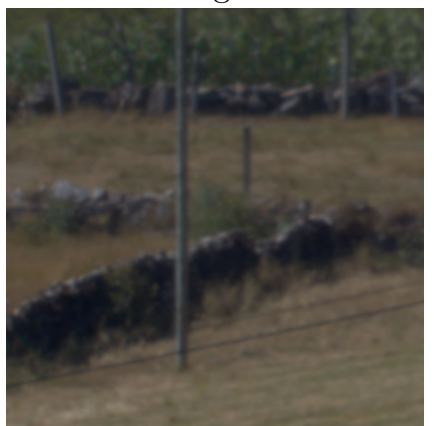

Image 5

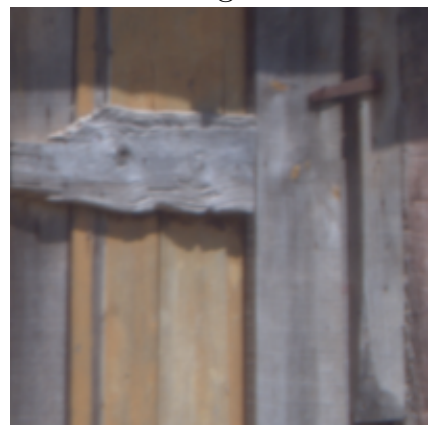

Image 8

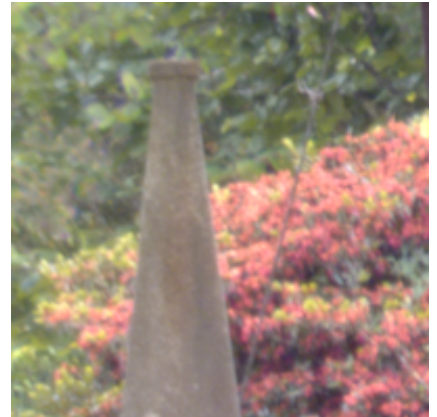

Image 3

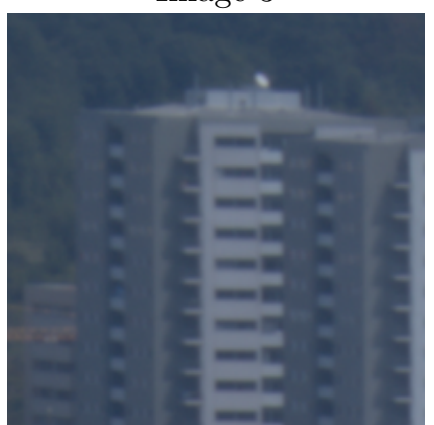

Image 6

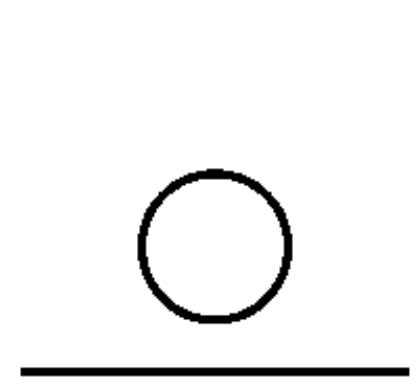

Image 9

Figure 3: Test images

e) The utilisation of diagonally connected pixels is favourable to the vector median in case of large neighbourhoods;

f) The freedom from the necessity of forming pseudo-pixels passing through the centre in the vicinity improves the performance of the vector median in particular in case of large neighbourhood.

Furthermore, PSNRs shown in Table 2 are mostly slightly higher in comparison with those in Table 1.

A few pictorial results were demonstrated in Figure 6-9 for the sake of visual comparison. Demonstrated results of vector median was computed within large neighbourhood where passing centre pixel was not a necessity and diagonally connected pixels were not considered for the formation of pseudo-pixels. The images basically correspond to the numerical results shown before, although the influence of the refinement is not visible enough.

\section{CONCLUSION}

In this paper two vector based median filtering techniques were presented, namely the vector median for MSFA demosaicking and median in n-sphere. In order to evaluate these methods, a system capable of MSFA mosaicking 

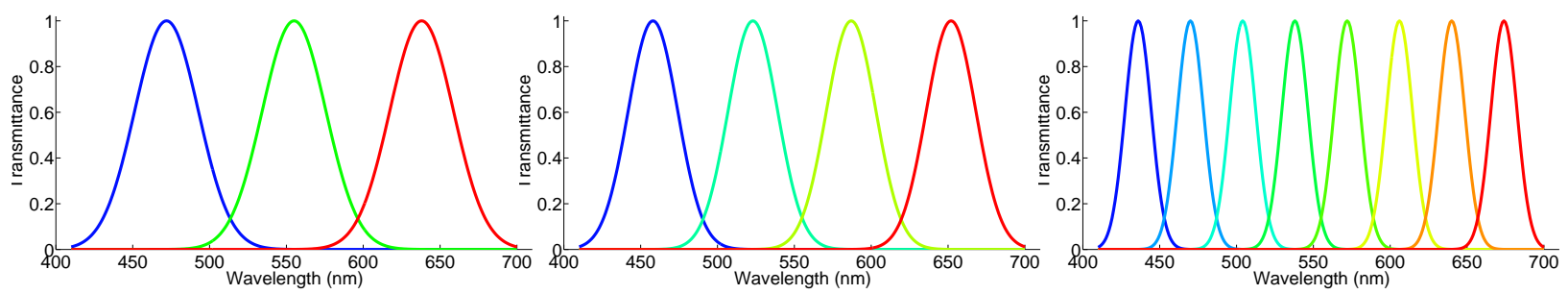

Figure 4: Filter design for 3-band, 4-band and 8-band set-up
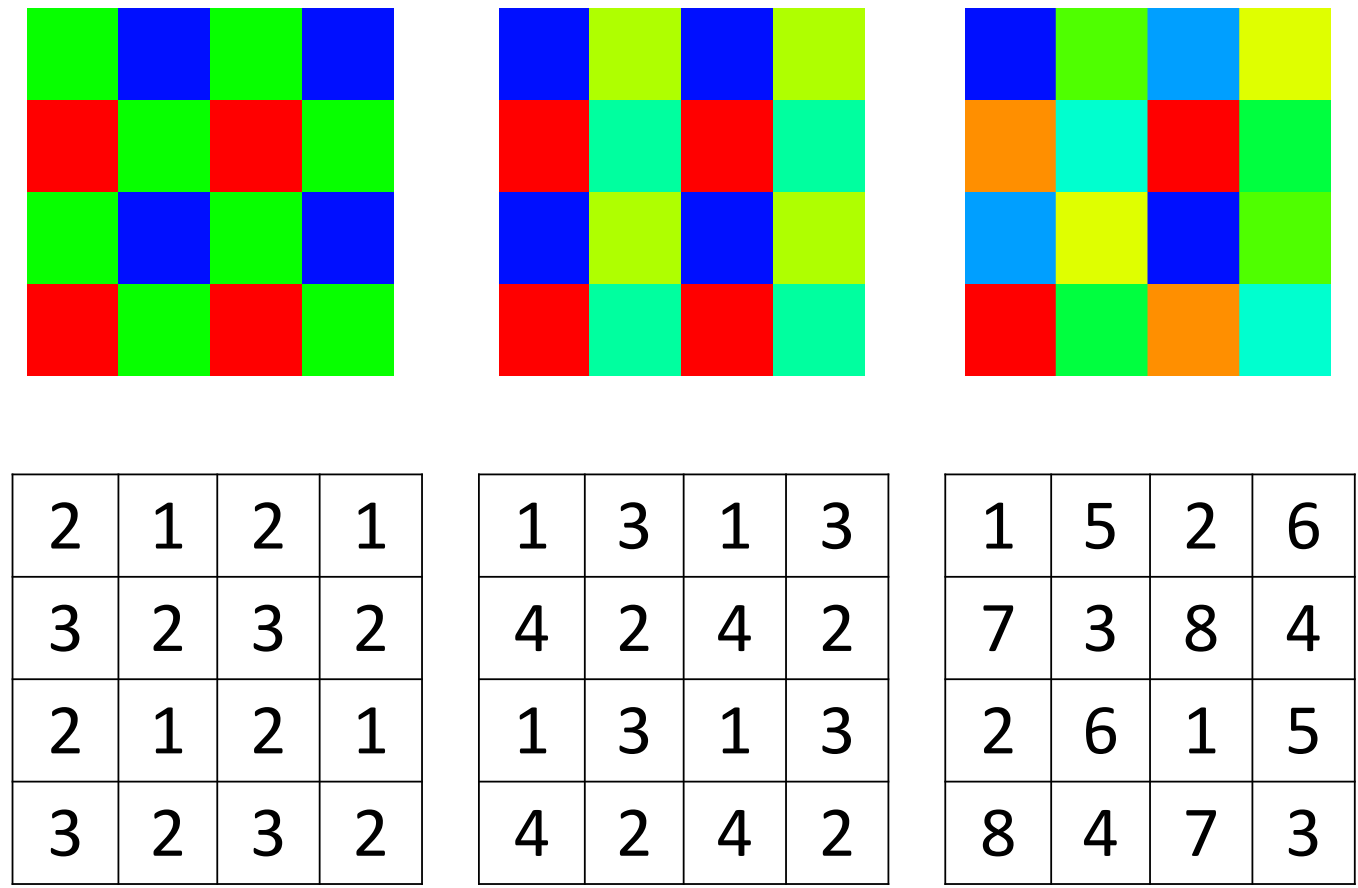

Figure 5: MSFA design for 3-band, 4-band and 8-band set-up

and demosaicking was developed.

As can be seen from the results, vector median filters perform reasonably in extreme conditions as is the case of binary images. Otherwise it does not behave as well as bilinear and binary tree approaches, contrary to expectations. It is somewhat surprising that the authors failed to reproduce similar results to those shown by the original work. ${ }^{5}$ The results also indicate that it is necessary that more than one pixel of a certain spectral band occur in the vicinity where pseudo-pixels are formed.

It is of importance that in most cases median filtering in n-sphere reduced to some extent the disparity between original and demosaicked images in terms of PSNR, and the improvement coincided with visual judgements in that less aliasing appeared in refined images. This proved that the advantages of median filtering in angular space still holds in multispectral domain. However, the impact of variable filter window size, remained to be seen.

Another point of particular interest is that none of the demosaicking algorithms experimented within this paper took advantage of inter-channel correlation, whereas this is one of the basic premises of most CFA demosaicking techniques. The correlation could have been employed more efficiently in the preceding demosaicking process. Some measures are expected later to be introduced in this regard. 
Table 1: The performance of the demosaicking algorithms. sNB/lNB denote small/large neighbourhood, PC/nPC indicates whether the pseudo-pixels have to pass the central pixel, HV/DIAG signifies whether the pseudo-pixels are formed by horizontally and vertically connected pixels only or diagonally connected pixels are considered as well.

\begin{tabular}{|c|c|c|c|c|c|c|c|c|c|c|}
\hline \multirow{2}{*}{ MSFA } & \multirow{2}{*}{ Methods } & \multicolumn{9}{|c|}{ Image } \\
\hline & & 1 & 2 & 3 & 4 & 5 & 6 & 7 & 8 & 9 \\
\hline \multirow{3}{*}{ 3-band } & Bilinear & 41.29 & 41.20 & 28.23 & 44.03 & 47.08 & 46.79 & 31.38 & 40.09 & 18.11 \\
\hline & Binary Tree & 41.98 & 43.61 & 28.60 & 46.21 & 47.39 & 50.35 & 33.11 & 41.47 & 19.58 \\
\hline & VM & 35.87 & 36.89 & 23.60 & 39.43 & 42.12 & 40.65 & 27.04 & 35.73 & 28.50 \\
\hline \multirow{7}{*}{ 4-band } & Bilinear & 38.46 & 39.85 & 26.80 & 41.49 & 44.97 & 45.33 & 29.95 & 38.48 & 16.63 \\
\hline & Binary Tree & 38.19 & 40.01 & 26.23 & 42.55 & 44.44 & 47.11 & 30.02 & 38.76 & 16.95 \\
\hline & $\mathrm{VM}(\mathrm{sNB} / \mathrm{PC} / \mathrm{HV})$ & 32.62 & 33.50 & 21.75 & 34.65 & 39.39 & 36.51 & 22.76 & 31.70 & 14.38 \\
\hline & $\mathrm{VM}(\mathrm{sNB} / \mathrm{PC} / \mathrm{DIAG})$ & 32.84 & 33.41 & 21.76 & 34.60 & 39.50 & 36.60 & 22.91 & 31.84 & 13.88 \\
\hline & $\mathrm{VM}(\mathrm{lNB} / \mathrm{PC} / \mathrm{HV})$ & 31.00 & 31.56 & 19.44 & 33.53 & 37.14 & 35.54 & 22.32 & 30.62 & 13.23 \\
\hline & $\mathrm{VM}(\mathrm{lNB} / \mathrm{nPC} / \mathrm{HV})$ & 33.60 & 34.77 & 22.39 & 36.94 & 40.61 & 38.52 & 25.22 & 33.94 & 31.94 \\
\hline & $\mathrm{VM}(\mathrm{lNB} / \mathrm{PC} / \mathrm{DIAG})$ & 31.25 & 31.99 & 19.83 & 34.12 & 37.51 & 35.97 & 22.48 & 31.02 & 13.35 \\
\hline \multirow{8}{*}{ 8-band } & Bilinear & 31.55 & 30.87 & 18.70 & 33.77 & 36.88 & 35.87 & 21.08 & 31.07 & 11.63 \\
\hline & Binary Tree & 31.04 & 32.00 & 19.22 & 35.37 & 38.07 & 39.63 & 25.66 & 33.12 & 13.20 \\
\hline & $\mathrm{VM}(\mathrm{sNB} / \mathrm{PC} / \mathrm{HV})$ & 29.31 & 29.82 & 17.93 & 31.82 & 36.34 & 34.26 & 21.18 & 29.82 & 16.93 \\
\hline & $\mathrm{VM}(\mathrm{sNB} / \mathrm{nPC} / \mathrm{HV})$ & 28.19 & 28.87 & 16.95 & 31.61 & 34.95 & 33.43 & 19.77 & 28.93 & 20.87 \\
\hline & VM(sNB/PC/DIAG) & 29.05 & 29.58 & 17.68 & 31.83 & 35.81 & 33.67 & 19.98 & 29.14 & 17.31 \\
\hline & $\mathrm{VM}(\mathrm{lNB} / \mathrm{PC} / \mathrm{HV})$ & 26.35 & 26.55 & 15.00 & 28.95 & 32.89 & 31.04 & 17.54 & $26.4^{2}$ & 8.60 \\
\hline & $\mathrm{VM}(\mathrm{lNB} / \mathrm{nPC} / \mathrm{HV})$ & 26.26 & 27.33 & 15.50 & 30.59 & 33.56 & 31.86 & 18.19 & 27.80 & 17.33 \\
\hline & $\mathrm{VM}(\mathrm{lNB} / \mathrm{PC} / \mathrm{DIAG})$ & 26.90 & 27.19 & 15.56 & 29.88 & 33.49 & 31.73 & 18.23 & 27.25 & 9.20 \\
\hline
\end{tabular}

Table 2: The performance of demosaicking algorithms after refinement

\begin{tabular}{|c|c|c|c|c|c|c|c|c|c|c|}
\hline \multirow{2}{*}{ MSFA } & \multirow{2}{*}{ Methods } & \multicolumn{9}{|c|}{ Image } \\
\hline & & 1 & 2 & 3 & 4 & 5 & 6 & 7 & 8 & 9 \\
\hline \multirow{3}{*}{ 3-band } & Bilinear & 41.72 & 41.09 & 27.36 & 43.64 & 47.88 & 47.47 & 32.87 & 41.71 & 19.35 \\
\hline & Binary Tree & 41.93 & 42.59 & 27.41 & 44.97 & 47.83 & 49.68 & 34.60 & 42.72 & 20.71 \\
\hline & VM & 36.72 & 36.46 & 23.41 & 36.64 & 41.21 & 39.86 & 27.12 & 37.22 & 23.18 \\
\hline \multirow{7}{*}{ 4-band } & Bilinear & 38.98 & 39.73 & 26.26 & 41.10 & 45.85 & 45.79 & 31.39 & 39.86 & 17.70 \\
\hline & Binary Tree & 38.39 & 39.57 & 25.60 & 41.68 & 44.96 & 46.80 & 31.20 & 39.66 & 17.62 \\
\hline & $\mathrm{VM}(\mathrm{sNB} / \mathrm{PC} / \mathrm{HV})$ & 34.23 & 35.20 & 22.70 & 36.28 & 41.32 & 38.73 & 24.91 & 33.95 & 16.33 \\
\hline & $\mathrm{VM}(\mathrm{sNB} / \mathrm{PC} / \mathrm{DIAG})$ & 35.65 & 35.97 & 23.16 & 37.20 & 42.58 & 40.59 & 26.41 & 35.59 & 15.81 \\
\hline & $\mathrm{VM}(\mathrm{lNB} / \mathrm{PC} / \mathrm{HV})$ & 31.91 & 32.36 & 19.85 & 34.50 & 38.01 & 37.15 & 24.03 & 32.03 & 14.48 \\
\hline & $\mathrm{VM}(\mathrm{lNB} / \mathrm{nPC} / \mathrm{HV})$ & 34.36 & 35.41 & 22.44 & 37.90 & 41.47 & 40.03 & 26.60 & 35.45 & 31.94 \\
\hline & $\mathrm{VM}(\mathrm{lNB} / \mathrm{PC} / \mathrm{DIAG})$ & 32.07 & 32.71 & 20.17 & 35.11 & 38.33 & 37.51 & 24.15 & 32.37 & 14.73 \\
\hline \multirow{8}{*}{ 8-band } & Bilinear & 32.51 & 31.79 & 19.55 & 34.32 & 38.15 & 37.04 & 22.64 & 32.49 & 12.49 \\
\hline & Binary Tree & 31.41 & 32.27 & 19.44 & 35.31 & 38.50 & 39.77 & 26.27 & 33.69 & 13.62 \\
\hline & $\mathrm{VM}(\mathrm{sNB} / \mathrm{PC} / \mathrm{HV})$ & 30.83 & 31.34 & 19.14 & 33.29 & 37.99 & 36.08 & 23.32 & 31.78 & 18.73 \\
\hline & $\mathrm{VM}(\mathrm{sNB} / \mathrm{nPC} / \mathrm{HV})$ & 28.91 & 29.72 & 17.49 & 32.80 & 35.73 & 34.69 & 21.01 & 30.12 & 22.04 \\
\hline & $\mathrm{VM}(\mathrm{sNB} / \mathrm{PC} / \mathrm{DIAG})$ & 30.32 & 30.84 & 18.47 & 33.42 & 37.10 & 36.14 & 21.93 & 31.35 & 18.99 \\
\hline & $\mathrm{VM}(\mathrm{lNB} / \mathrm{PC} / \mathrm{HV})$ & 27.10 & 27.25 & 15.66 & 29.78 & 33.57 & 31.98 & 18.43 & 27.28 & 8.81 \\
\hline & $\mathrm{VM}(\mathrm{lNB} / \mathrm{nPC} / \mathrm{HV})$ & 26.56 & 27.71 & 15.72 & 31.20 & 33.90 & 32.49 & 18.67 & 28.40 & 17.33 \\
\hline & $\mathrm{VM}(\mathrm{lNB} / \mathrm{PC} / \mathrm{DIAG})$ & 27.62 & 27.82 & 16.04 & 30.67 & 34.11 & 32.75 & 19.13 & 28.17 & 9.27 \\
\hline
\end{tabular}

In conclusion, we observed that increased number of spectral channels reduced significantly spatial resolution of the image and reproduced image quality suffered in consequence, which deserves further consideration. The results showed that median filtering in n-sphere (n-dimensional spherical space) reduces aliasing numerically for most test images, thus improving resulting image quality. Also the results have initially demonstrated the validity of our assumption that certain demosaicking algorithms developed for trichromatic images may be useful for MSFA demosaicking purpose. In addition, some image quality metrics specific to MSFA demosaicking is reasonably expected. 


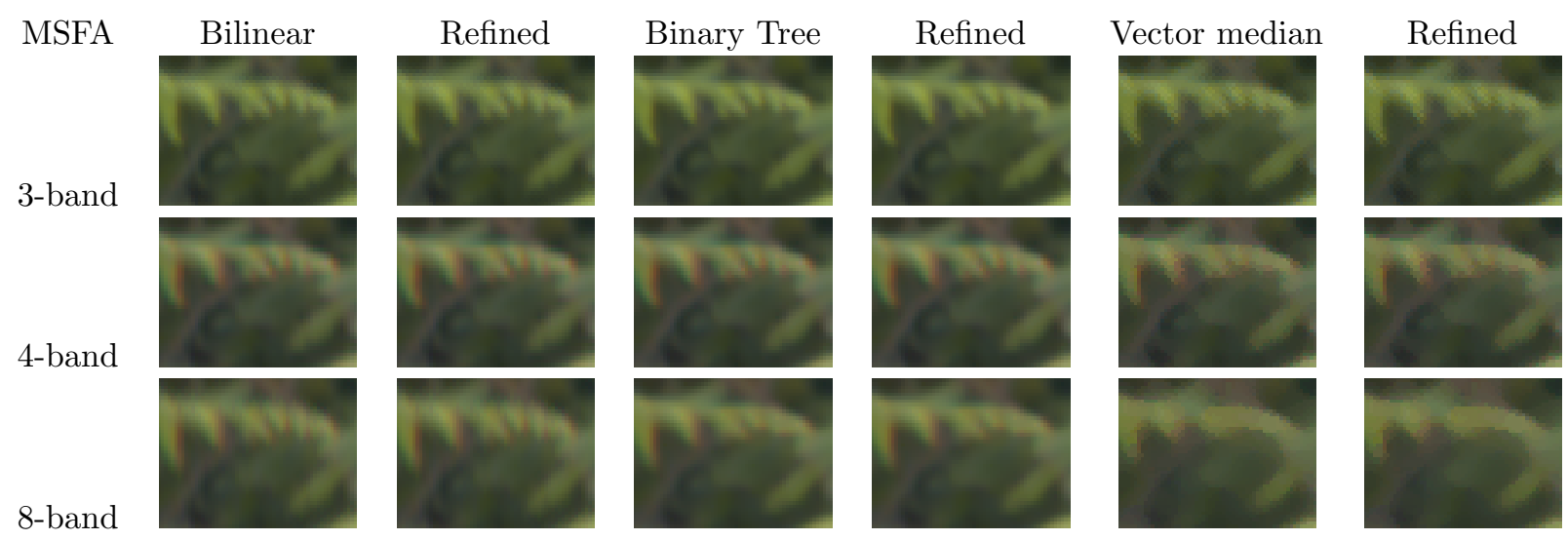

Figure 6: An example of Image 2
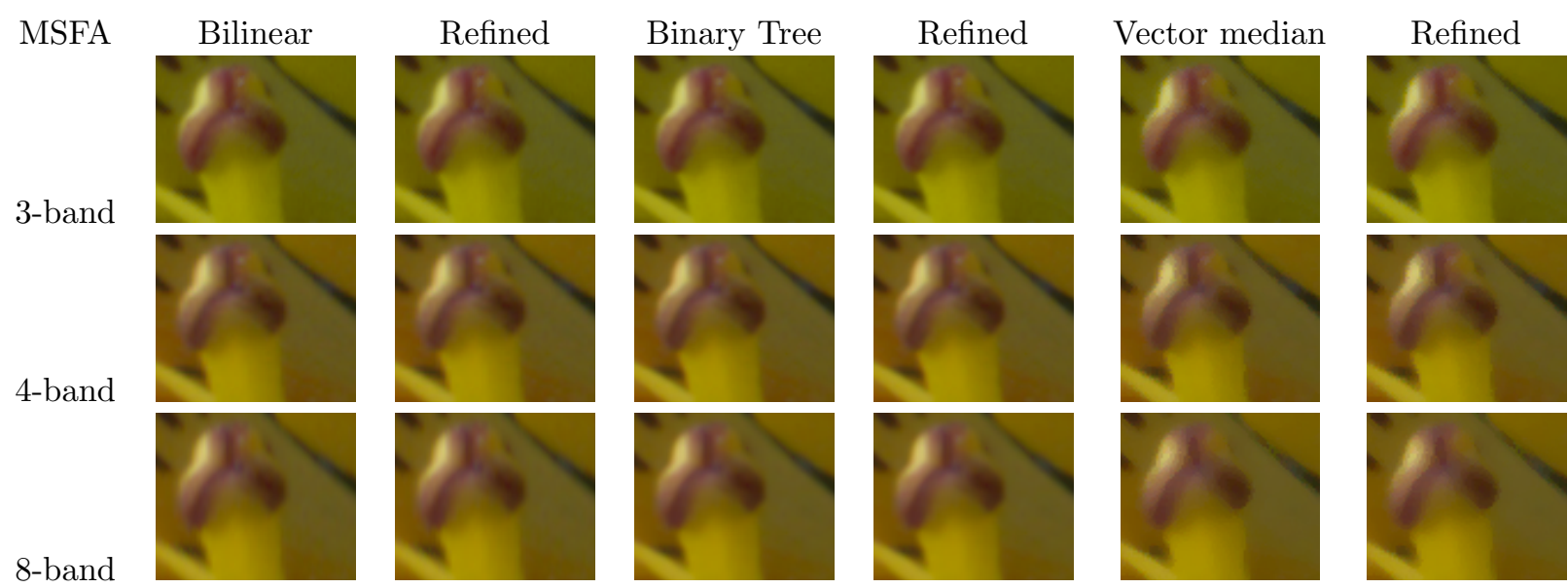

Figure 7: An example of Image 4
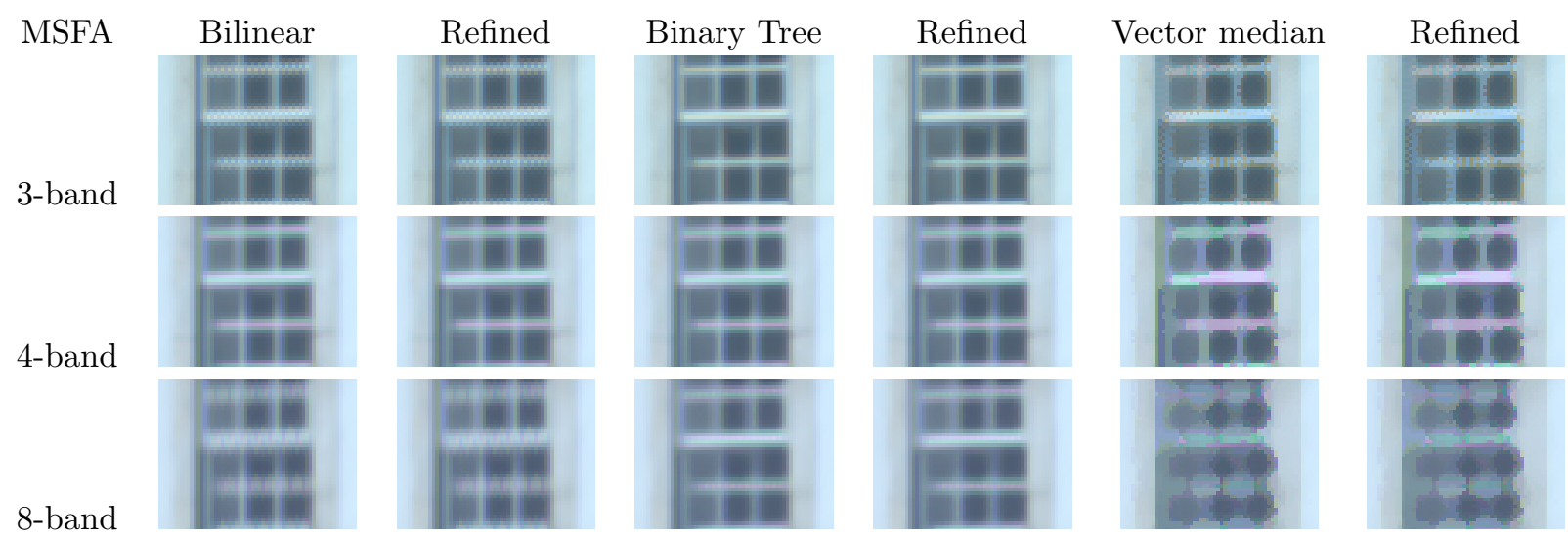

Figure 8: An example of Image 7

\section{ACKNOWLEDGMENTS}

This work is jointly funded by the regional council of Burgundy in France and Gjøvik University College in Norway. The authors are grateful to Prof. Ivar Farup of Gjøvik University College for the fruitful discussion. 


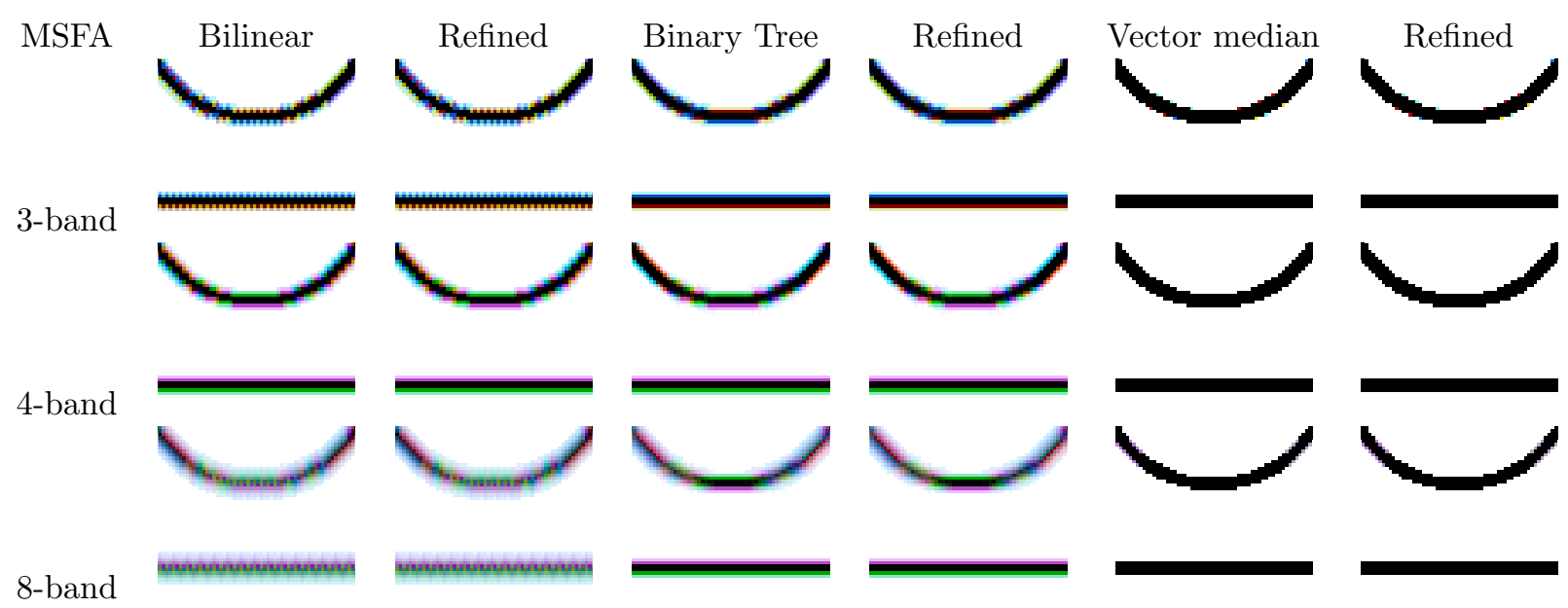

Figure 9: An example of Image 9

\section{REFERENCES}

[1] Bayer, B. E., "Color imaging array." Patent (07 1976). US 3971065.

[2] Miao, L., Qi, H., and Snyder, W. E., "A generic method for generating multispectral filter arrays," in [International Conference on Image Processing], 5, 3343 - 3346 (Oct. 2004).

[3] Miao, L., Qi, H., and Ramanath, R., "A generic binary tree-based progressive demosaicking method for multispectral filter array," in [IEEE International Conference on Image Processing], 3221 -3224 (Oct. 2006).

[4] Shrestha, R., Hardeberg, J. Y., and Khan, R., "Spatial arrangement of color filter array for multispectral image acquisition," in [Sensors, Cameras, and Systems for Industrial, Scientific, and Consumer Applications XII], Widenhorn, R. and Nguyen, V., eds., Proc. SPIE 7875, 787503-787503-9, SPIE (Jan. 2011).

[5] Gupta, M. R. and Chen, T., "Vector color filter array demosaicing," in [Sensors and Camera Systems for Scientific, Industrial, and Digital Photography Applications II], Blouke, M. M., Canosa, J., and Sampat, N., eds., Proc. SPIE 4306, 374-382, SPIE (May 2001).

[6] Keren, D. and Osadchy, M., "Restoring subsampled color images," Machine Vision and Applications 11, 197-202 (Dec. 1999).

[7] Astola, J., Haavisto, P., and Neuvo, Y., "Vector median filters," Proceedings of the IEEE 78, 678 -689 (Apr. 1990).

[8] Foster, D. H., Amano, K., Nascimento, S. M. C., and Foster, M. J., "Frequency of metamerism in natural scenes," J. Opt. Soc. Am. A 23, 2359-2372 (Oct 2006).

[9] Hardeberg, J. Y., [Acquisition and Reproduction of Color Images: Colorimetric and Multispectral Approaches], dissertation.com. (2001). 